12,05

\title{
Механизм открытия щели в точке Дирака в электронном спектре Gd-допированного топологического изолятора
}

\author{
() А.М. Шикин, Д.А. Естюнин, А.В. Королева, Д.А. Глазкова, Т.П. Макарова, С.О. Фильнов \\ Санкт-Петербургский государственный университет, \\ Санкт-Петербург, Россия \\ E-mail: ashikin@inbox.ru
}

Поступила в Редакцию 24 сентября 2019 г.

В окончательной редакции 22 октября 2019 г.

Принята к публикации 23 октября 2019 г.

Методами фотоэлектронной спектроскопии с угловым разрешением проведены исследования электронной структуры в области точки Дирака для магнитно-допированного топологического изолятора $\mathrm{Bi}_{1.09} \mathrm{Gd}_{0.06} \mathrm{Sb}_{0.85} \mathrm{Te}_{3}$ при различных температурах (выше и ниже температуры Нееля, $1-35 \mathrm{~K}$ ) и поляризациях синхротронного излучения. Показано наличие энергетической щели в точке Дирака, формируемой в фотоэмиссионных спектрах, которая остается открытой выше температуры дальнодействующего магнитного упорядочения, $T_{H}$. Измерения магнитных свойств методом сверхпроводящей магнитометрии показали антиферромагнитное упорядочение с температурой перехода в парамагнитную фазу, равной $8.3 \mathrm{~K}$. Исследования температурной зависимости интенсивности состояний конуса Дирака методом фотоэлектронной спектроскопии подтвердили наличие магнитного перехода и показали возможность его индикции непосредственно из фотоэмиссионных спектров. Более детальный анализ величины расщепления между состояниями верхнего и нижнего конусов Дирака (т. е. величины энергетической щели) в точке Дирака в фотоэлектронных спектрах показал зависимость щели в точке Дирака от типа поляризации синхротронного излучения $(28-30 \mathrm{meV}$ для p-поляризации и $22-25 \mathrm{meV}$ для циркулярно-поляризованного излучения противоположной хиральности). В работе предложен механизм открытия щели в точке Дирака выше $T_{H}$ вследствие „спаривания“ дираковских фермионов с противоположным импульсом и спиновой ориентацией в результате их взаимодействия со спиновой текстурой, формируемой непосредственно в процессе фотоэмиссии в области фотоэмиссионной дырки на атоме магнитной примеси $(\mathrm{Gd})$. Было показано, что щель в точке Дирака, измеряемая выше $T_{H}$ является динамической и формируется непосредственно в процессе фотоэмиссии. При этом природа щели остается магнитной (даже при отсутствии дальнодействующего магнитного упорядочения) и обусловлена свойствами магнитного топологического изолятора, что и определяет практически неизменность величины щели при переходе через $T_{H}$. Подтверждением динамического характера генерируемой щели является зависимость ее величины от поляризации синхротронного излучения.

Ключевые слова: антиферромагнитный топологический изолятор, фотоэлектронная спектроскопия с угловым разрешением, фотоиндуцированная намагниченность.

DOI: 10.21883 /FTT.2020.02.48881.589

\section{1. Введение}

Взаимосвязь между топологией и магнетизмом играет важную роль в реализации ряда важных топологических квантовых эффектов, таких как топологический магнитоэлектрический эффект $[1,2]$ и квантовый аномальный эффект Холла (QАНЕ) [3,4], наблюдаемых в магнитных топологических изоляторах (ТИ). Данные эффекты являются важными для фундаментальной науки и могут быть использованы для развития будущих технологий, таких как бездиссипативная топологическая электроника и топологические квантовые вычисления. Наличие подобных эффектов основано на существовании топологических поверхностных состояний типа конуса Дирака с геликоидальной спиновой структурой и энергетической щелью в точке Дирака, открываемой за счет нарушения симметрии обращения времени. Одним из возможных способов нарушить симметрию обращения времени является легирование ТИ магнитными примесями. Для анализа электронной структуры в области энергетической щели в точке Дирака и ее зависимости от температуры и уровня магнитного легирования обычно используется метод фотоэлектронной (ФЭ) спектроскопии с угловым разрешением (ФЭСУР), см. например [5-17]. При этом зачастую оказывается, что измеряемые дисперсионные картины для ряда магнитных ТИ демонстрируют слабую зависимость от температуры. В измеряемых ФЭСУР-дисперсиях наблюдается сохранение щели в точке Дирака выше температуры Кюри или Нееля, для ферромагнитных $(\Phi \mathrm{M})$ и антиферромагнитных (АФМ) ТИ, т. е. при нарушении дальнодействующего магнитного упорядочения [7-9,11-17].

В литературе делались попытки объяснения слабой температурной зависимости щели в точке Дирака и ее наблюдения выше температуры магнитного упорядочения, например, в работах [7,14-20]. При этом, с одной стороны, высказывались предположения о немагнитной природе щели (за счет эффектов „непересечения“ элек- 
тронных состояний, открывающих гибридизационную немагнитную щель) [17-19] или повышенной поверхностной температуры Кюри [20]. С другой стороны, открытие щели связывалось с эффектами индуцированной намагниченности, обусловленной нескомпенсированной спиновой аккумуляцией и соответствующим спин-торк-эффектом, возникающих благодаря неэквивалентному фотовозбуждению электронов с противоположных ветвей дираковского конуса электронных состояний [7,14-16]. При этом была выявлена зависимость щели в точке Дирака, наблюдаемая в ФЭСУР от энергии фотонов и поляризации излучения, которые не могут быть объяснены в рамках перечисленных выше эффектов.

Данная работа посвящена анализу формирования энергетической щели, открываемой в точке Дирака для антиферромагнитного Gd-допированного ТИ со стехиометрий $\mathrm{Bi}_{1.09} \mathrm{Gd}_{0.06} \mathrm{Sb}_{0.85} \mathrm{Te}_{3}$ при различных температуpax. Данное соединение характеризуются локализацией точки Дирака в области уровня Ферми, что является необходимым условием для реализации QАНЕ [21]. Хорошо известно, что легирование магнитными примесями переходного металла, приводящее к открытию щели в точке Дирака, сопровождается также созданием примесной зоны в области уровня Ферми, вследствие несовпадения валентности Вi и переходного металла, атомы которого замещают атомы Ві при допировании [22]. Формируемые при этом состояния могут проводить зарядовые токи, которые шунтируют спиновые токи, необходимые для реализации QАНЕ. Однако, если легирующими магнитными примесями являются атомы редкоземельных металлов, такие как $\mathrm{Gd}$, который в данных соединениях имеет такую же валентность, как и $\mathrm{Bi}$, то формирование такой зоны примесных состояний вблизи уровня Ферми не предполагается [22]. К тому же, локализация точки Дирака вблизи уровня Ферми (при отсутствии других состояний) позволяет уменьшить влияние валентных состояний и зоны проводимости на формируемые магнитные свойства и усилить проявление магнитных топологических эффектов.

Основные усилия в данной работе были сосредоточены на анализе электронной структуры и формировании энергетической щели в области точки Дирака при различных температурах, в частности, при переходе через температуру объемного магнитного разупорядочения, температуру Нееля, $T_{H}$, (значение которой было определено из анализа магнитных свойств $\left.\mathrm{Bi}_{1.09} \mathrm{Gd}_{0.06} \mathrm{Sb}_{0.85} \mathrm{Te}_{3}\right)$. В работе будет показано, что щель в точке Дирака, измеряемая методом ФЭСУР, остается открытой и выше $T_{H}$. Будет предложен механизм формирования щели выше ТН за счет „спаривания“ дираковских фермионов посредством спиновой текстуры, генерируемой фотоэмиссией на атоме магнитной примеси. Динамический характер щели будет подтвержден зависимостью величины щели в точке Дирака от поляризации синхротронного излучения и спиновой поляризацией, генерируемой фотоэмиссией по нормали к поверхности.

\section{2. Результаты}

Для анализа магнитных свойств $\mathrm{Bi}_{1.09} \mathrm{Gd}_{0.06} \mathrm{Sb}_{0.85} \mathrm{Te}_{3}$ были проведены измерения индуцированной намагниченности в зависимости от приложенного магнитного поля и температуры с использованием сверхпроводящего квантового интерференционного магнитометра (СКВИД). На рис. 1, a показаны зависимости индуцированной намагниченности от магнитного поля, приложенного перпендикулярно поверхности, при различных температурах в диапазоне между 2 и $300 \mathrm{~K}$. Представленные экспериментальные зависимости демонстрируют $S$-образный ход индуцированной намагниченности с постепенным насыщением, которое достигается при приложенном внешнем магнитном поле 2-3 Т. Такое поведение является характерным для АФМ ТИ, допированных атомами редкоземельных (Р3М) металлов [5-8]. При температурах более $10 \mathrm{~K}$ объем образца переходит в парамагнитное (ПМ) состояние.

На рис. 1, $b$ показана температурная зависимость магнитной восприимчивости при приложении внешнего магнитного поля 5T, перпендикулярно поверхности, в диапазоне температур от 2 до $300 \mathrm{~K}$. Здесь же на вставке показана температурная зависимость величины обратной магнитной восприимчивости $1 /(\chi)$ с аппроксимацией зависимости законом Кюри-Вейсса $(\chi=C(T-\Theta)$, где $C-$ зависящая от материала постоянная Кюри, а $\Theta$ - температура Вейсса). Представленная аппроксимация (в линейном приближении зависимости $1 /(\chi))$ пересекает нулевой уровень при отрицательной температуре $(T=-8.3 \mathrm{~K})$, что свидетельствует об АФМ-упорядочении при низких температурах с температурой Нееля $\left(T_{H}\right)$ перехода из антиферромагнитного в парамагнитное состояние, равной $8.3 \mathrm{~K}$.

На рис. 2, $а$ представлены дисперсионные зависимости в виде $N(E)$ и $d^{2} N / d E^{2}$, измеренные методом ФЭСУР для $\mathrm{Bi}_{1.09} \mathrm{Gd}_{0.06} \mathrm{Sb}_{0.85} \mathrm{Te}_{3}$ при температуре $1 \mathrm{~K}$, значительно ниже наблюдаемой температуры Нееля. Вторая производная дисперсионной зависимости показана для большей детализации электронной структуры в области точки Дирака. Здесь же представлены профили спектров, измеренные непосредственно в точке Дирака (при $\left.k_{\text {II }}=0\right)$ и симметрично относительно точки Дирака при

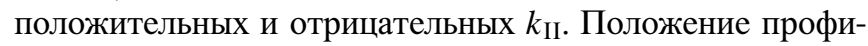
лей показано на панели с дисперсионной зависимостью соответствующими вертикальными цветными линиями. Для спектра, измеренного в точке Дирака, приведено разложение на спектральные составляющие, соответствующие максимумам плотности состояний верхнего и нижнего дираковского конуса на краях щели, открываемой в точке Дирака. Дисперсионная зависимость, представленная в форме $d^{2} N / d E^{2}$, а также разложение на спектральные составляющие ФЭ-спектра, измеренного 

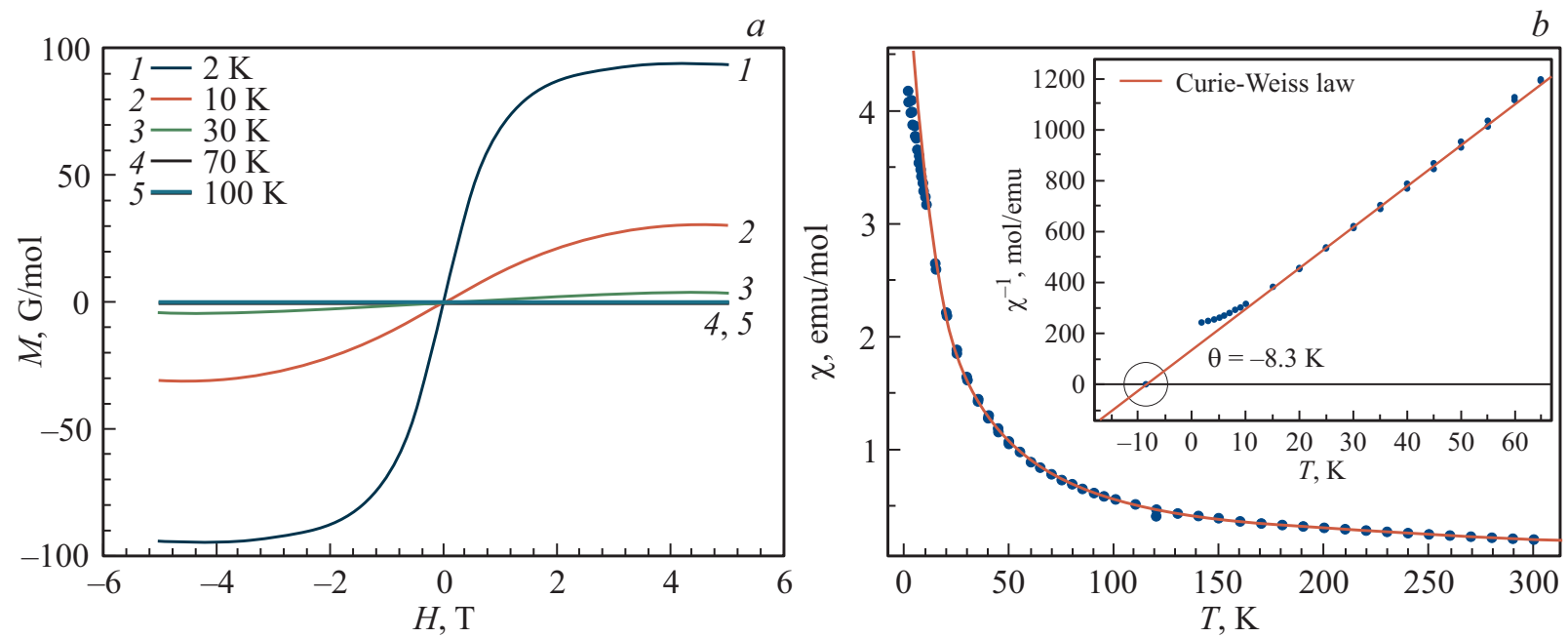

Рис. 1. $a-$ Зависимость индуцированной намагниченности от магнитного поля, приложенного перпендикулярно поверхности для $\mathrm{Bi}_{1.09} \mathrm{Gd}_{0.06} \mathrm{Sb}_{0.85} \mathrm{Te}_{3}$, методом СКВИД при различных температурах от 2 до $100 \mathrm{~K}$. $b-$ Температурная зависимость магнитной восприимчивости $(\chi)$, измеренная при приложении внешнего магнитного поля 5 Т, перпендикулярно поверхности, с аппроксимацией, рассчитанной по закону Кюри-Вейсса (сплошная линия).Вставка - температурная зависимость обратной магнитной восприимчивости $1 /(\chi)$ с соответствующим приближением при низких температурах (сплошная линия), показывающая отрицательное значение температуры $(\Theta=-8.3 \mathrm{~K})$.

при $k_{\mathrm{II}}=0$, показывают наличие энергетической щели в точке Дирака. Величина щели может быть оценена на уровне $35 \mathrm{meV}$. На панели справа представлена оценка величины расщепления между состояниями верхнего и нижнего дираковского конуса исходя из разложений фотоэмиссионных спектров (аналогично как для $k_{\mathrm{II}}=0$ ), при различных $k_{\text {II. Пунктирными линиями показана }}$ аппроксимация модельной функцией $\Delta \sim \sqrt{\alpha(k)^{2}+\lambda^{2}}$ данной зависимости, где $\lambda$ показывает величину запрещенной зоны, $\alpha-$ коэффициент пропорциональности для волнового вектора $k$. Такое дополнение позволяет значительно увеличить точность разложения.

При этом стоит отметить, что для данного соединения подобная оценка величины запрещенной зоны может оказаться завышенной вследствие вклада валентных состояний, расположенных в области нижнего конуса дираковских состояний, см. анализ ФЭ-спектров методом цикрулярного дихроизма ниже (рис. 4).

Для сравнительного анализа величины расщепления дираковских состояний при изменении энергетического положения точки Дирака, а также отстутвие влияния уровня Ферми на оценку запрещенной зоны, были измерены и проанализированы изменения дисперсионных зависимостей при напылении субмонослоя калия (около 0.3-0.5 монослоя). Напыление калия привело к сдвигу точки Дирака в сторону увеличения энергии связи на $0.1 \mathrm{eV}$ (рис. 2. $b$ ).

На рис. 2, $b$ представлены дисперсионные зависимости измеренные методом ФЭСУР при температуре $1.5 \mathrm{~K}$ и проведен анализ, подобный рис. 2, $a$, для системы $\mathrm{Bi}_{1.09} \mathrm{Gd}_{0.06} \mathrm{Sb}_{0.85} \mathrm{Te}_{3}$ с напылением субмонослоя калия. Несмотря на немного худшую статистику и энергетическое разрешение представленные зависимости также по- казывают величину расщепления дираковских состояний верхнего и нижнего конуса на уровне $35 \mathrm{meV}$. Из анализа представленных данных можно сделать важный вывод о том, что величина расщепления между состояниями верхнего и нижнего дираковских конусов в точке Дирака практически не зависит от энергетического положения точки Дирака относительно уровня Ферми.

Для более точного анализа зависимости величины расщепления состояний в точке Дирака от температуры был произведен непосредственный сравнительный анализ изменений интегральной ширины пика дираковских состояний в Г-точке в ФЭСУР спектрах при непрерывном повышении температуры от 1.5 до $30 \mathrm{~K}$, т.е. при переходе через $T_{H}$ (которая по данным, представленным на рис. 1 , составляет $8.3 \mathrm{~K}$ ). Все остальные параметры записи ФЭ-спектров при этом оставались без каких-либо изменений. На рис. 2,c представлены профили в Г точке ФЭСУР-спектров в области энергий связи $0-0.3 \mathrm{eV}$, включающей область пика дираковских состояний $(0.1-0.14 \mathrm{eV})$, при перманентном повышении температуры от 1.5 до $30 \mathrm{~K}$. Данный энергетический интервал соответствует области расщепления дираковских состояний в точке Дирака. Из анализа представленных температурных зависимостей видно, что ширина пика в области точки Дирака при переходе через $T_{H}$ (т.е. температуру дальнодействующего магнитного разупорядочения) остается практически неизменной. Меняется только интенсивность пика дираковсих состояний, чья температурная зависимость представлена на рис. 2, $d$. При этом в области температур ниже $T_{H}$ интенсивность пика дираковских состояний уменьшается с температурой, а при превышении $T_{H}$ остается практически неизменной. Область перехода через $T_{H}$ на наблюдаемой 

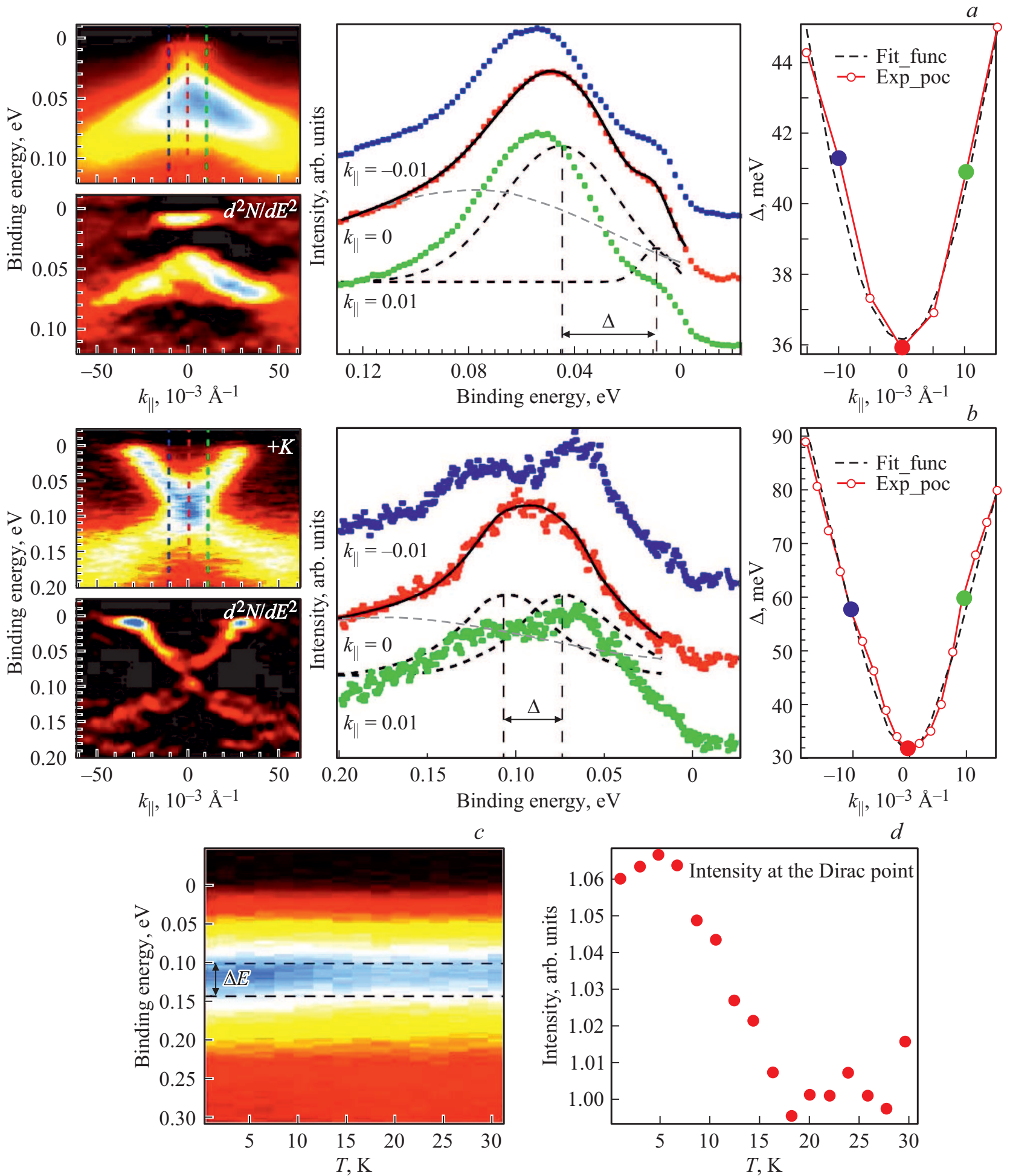

Рис. 2. $a$ - Дисперсионные ФЭСУР-зависимости в форме $N(E)$ и $d^{2} N / d E^{2}$, измеренные для $\mathrm{Bi}_{1.09} \mathrm{Gd}_{0.06} \mathrm{Sb}_{0.85} \mathrm{Te}_{3}$ ниже $T_{H}$ при температуре $1.5 \mathrm{~K}$. Здесь же представлены профили ФЭСУР-спектров, измеренные непосредственно в $\Gamma$-точке при $k_{\mathrm{II}}=0\left(\mathrm{~A}^{-1}\right)$

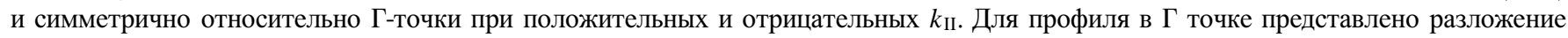
на спектральные составляющие.На правой панели показана величина расщепления между состояниями верхнего и нижнего дираковского конуса исходя из разложений профилей при различных $k_{\text {II }}$ на спектральные составляющие. $b-$ То же самое для $\mathrm{Bi}_{1.09} \mathrm{Gd}_{0.06} \mathrm{Sb}_{0.85} \mathrm{Te}_{3}$ с напыленным субмонослоем калия. $c, d-$ непрерывные температурные изменения интенсивности состояний в ФЭ-спектрах в области точки Дирака в виде профилей ФЭСУР-спектров в точке $\Gamma-c$, а также интегрированной интенсивности в области энергий связи $\Delta E=[0.1-0.14] \mathrm{eV}$ в диапазоне температур от 1 до $35 \mathrm{~K}-d$. 

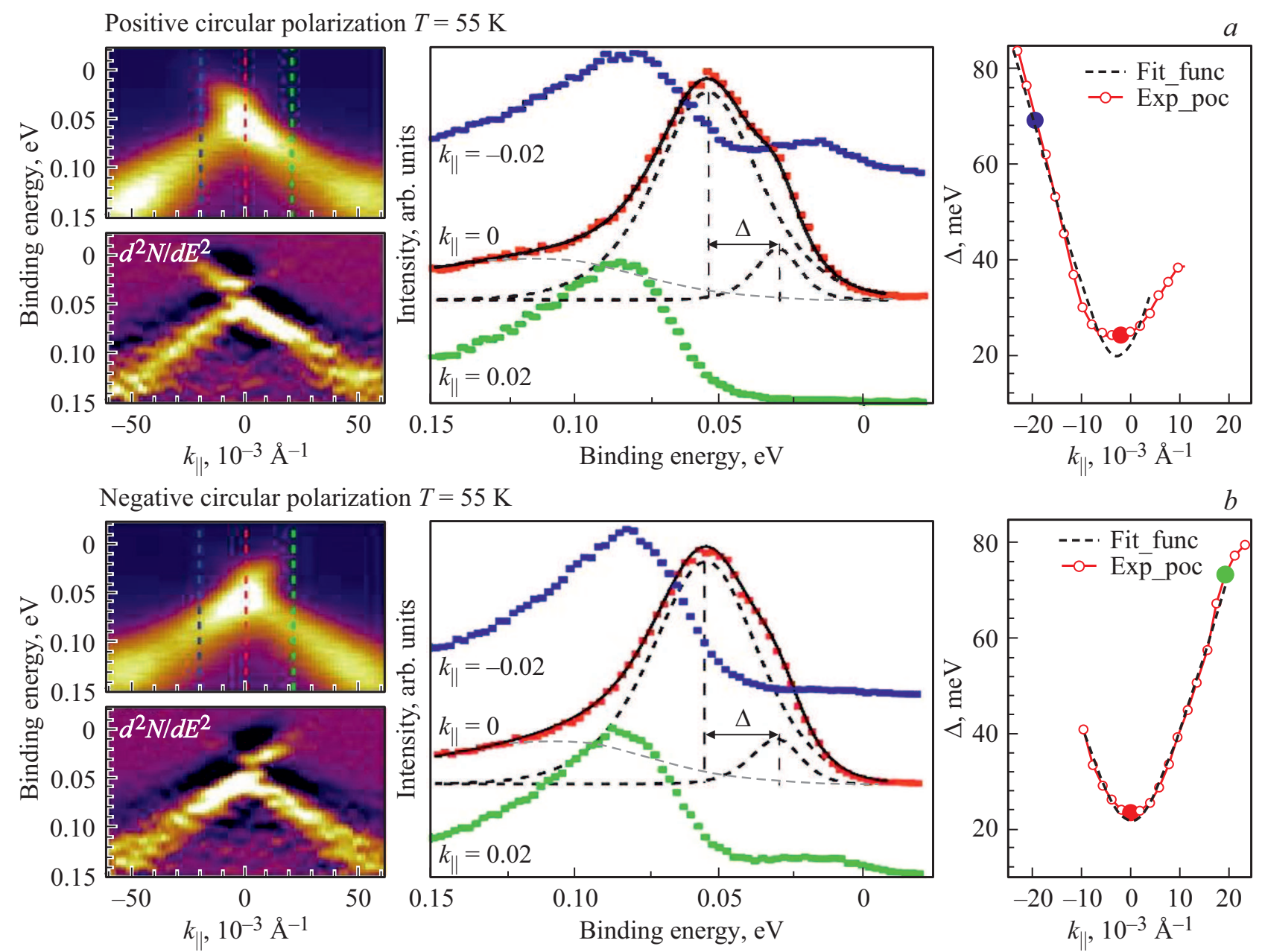

Рис. 3. Дисперсии дираковских поверхностных состояний, измеренные методом ФЭСУР (в форме $N(E)$ и $\left.d^{2} N / d E^{2}\right)$ для $\mathrm{Bi}_{1.09} \mathrm{Gd}_{0.06} \mathrm{Sb}_{0.85} \mathrm{Te}_{3}$ при температурах $55 \mathrm{~K}(a, b, c)$ и $17(d)$ с использованием СИ различной поляризации с энергий $28 \mathrm{eV}-$ левый столбец.Средний столбец - профили ФЭСУР-спектров, измеренные непосредственно в $\Gamma$-точке $\left(k_{\mathrm{II}}=0\right)$ и симметрично

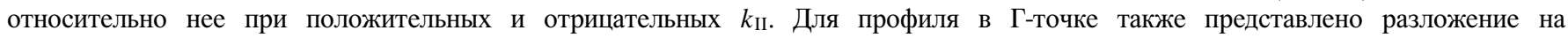
спектральные компоненты. Правая колонка - оценка величины расщепления между состояниями верхнего и нижнего конусов Дирака исходя из разложений ФЭ-спектров при различных $k_{\text {II }}$ на спектральные составляющие. Пунктирная линия показывает аппроксимацию зависимости расщепления модельной функций $\Delta$.

зависимости интенсивности пика дираковских состояний (рис. $2, d$ ) соответствует области температур 10-15K, что немного превышает температуру перехода полученную при помощи СКВИД магнитометрии. Расхождение температуры перехода оцененной методом СКВИД и из анализа интенсивности пиков дираковских состояний может быть объяснено наличием спиновых флуктуаций, подобно пленкам $\mathrm{Gd}$ и $\mathrm{Tb}[23,24]$, а также в системе $\mathrm{EuSi}_{2} \mathrm{Te}_{2}$ [25]. Подобное изменение является важным свидетельством наличия взаимодействия между электронной структурой дираковских состояний и магнетизмом в системе и, соответственно, возможной магнитной природе наблюдаемой запрещенной зоной в точке Дирака.

Для анализа величины расщепления между верхним и нижним конусом Дираковских состояний на рис. 3, $a, b, c$ представлены дисперсионные зависимости, измеренные методом ФЭСУР, для температуры $T=55 \mathrm{~K}$, что значительно выше $T_{H}$, для которых проведен анализ, подобный, как на рис. 2, $a, b$. Спектры измерены при энергии фотонов $28 \mathrm{eV}$ при использовании циркулярной поляризации СИ двух противоположных направлений $(a, b)$ и $p$-поляризации - $(c)$. На рис. $3, d$ представлены соответствующие дисперсионные зависимости и анализ ФЭСУР спектров, измеренных при температуре $17 \mathrm{~K}$ и p-поляризации СИ.

Результаты оценки величины расщепления дираковских состояний в точке Дирака с использованием ранее представленных методов (исходя из разложения на спектральные составляющие и из аппроксимации дисперсионных зависимостей) показывают величину расщепления состояний в точке Дирака около $28 \mathrm{meV}$ для $p$-поляризованного СИ. Аналогичные оценки для циркулярно-поляризованного СИ противоположной хи- 

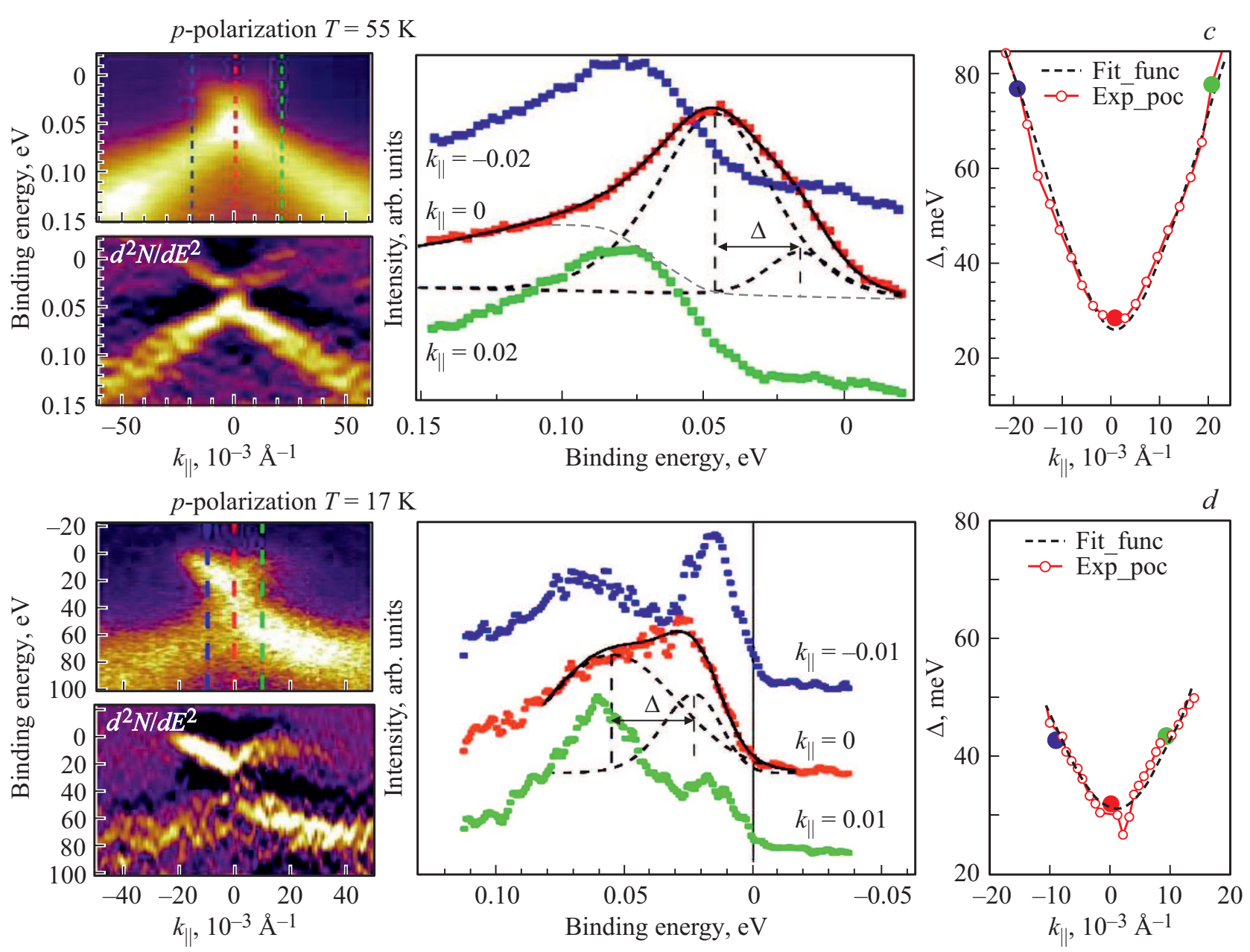

Рис. 3 (продолжение).

ральности дают величины $21-24 \mathrm{meV}$. Погрешность оценки величины запрещенной зоны в точке Дирака можно оценить на уровне $3-5 \mathrm{meV}$. Оценка величины расщепления для дисперсионных зависимостей, представленных на рис. 3, $d$ для температуры $17 \mathrm{~K}$ дает величину около $30-32 \mathrm{meV}( \pm 3-4 \mathrm{meV})$.

Данные измерения показывают несколько меньшую величину расщепления дираковских состояний в точке Дирака по сравнению с рис. 2, однако и в этом случае величина расщепления несколько завышена вследствие влияния спектрального веса вклада состояний валентной зоны, которые расположены в области нижнего конуса дираковских состояний. На рис. 4, $a$ показан циркулярный дихроизм для ФЭСУР-спектров представленных на рис. 3, $a, b$. Цветами различной тональности (соответствующими красному и синему цвету в оригинале) показаны вклады состояний, превалирующие при противоположных циркулярных поляризациях СИ, которые являются отображением спиновой структуры. Представленые дисперсии явным образом показывают локализацию состояний верхнего и нижнего конуса дираковских состояний (с противоположной спиновой ориентацией) и локализацию точки Дирака при энергии связи около
$0.05 \mathrm{eV}$. Рис. 4, $b$ подтверждает инверсию состояний дираковского конуса в области точки Дирака. С другой стороны, на представленных дисперсиях явно видно, что нижний конус дираковских состояний пересекается с состояниями валентной зоны, верний край которых уже явно виден начиная с энергий связи $0.08-0.09 \mathrm{eV}$. Ввиду большой интенсивности валентных состояний результирующий максимум дираковских состояний может сдвигаться в сторону более высоких энергий связи, визуально приводя к увеличению измеряемой фотоэмиссией величины расщепления дираковских состояний. Однако данная неопределенность экспериментальной оценки величины щели в точке Дирака (некоторое возможное ее завышение) не вляиет на основное заключение из анализа температурных зависимостей о том, что щель в точке Дирака остается открытой и выше $T_{H}$, практически не изменяясь по величине при переходе через $T_{H}$.

На рис. 5, а представлен ФЭ-спектр со спиновым разрешением для состояний Дираковского конуса, измеренный в точке Г для спиновой ориентации по нормали к поверхности при температуре $46 \mathrm{~K}$, что значительно выше $T_{H}$. Спектр измерен при энергии фотонов $28 \mathrm{eV}$ с использованием $p$-поляризованного синхротронного 

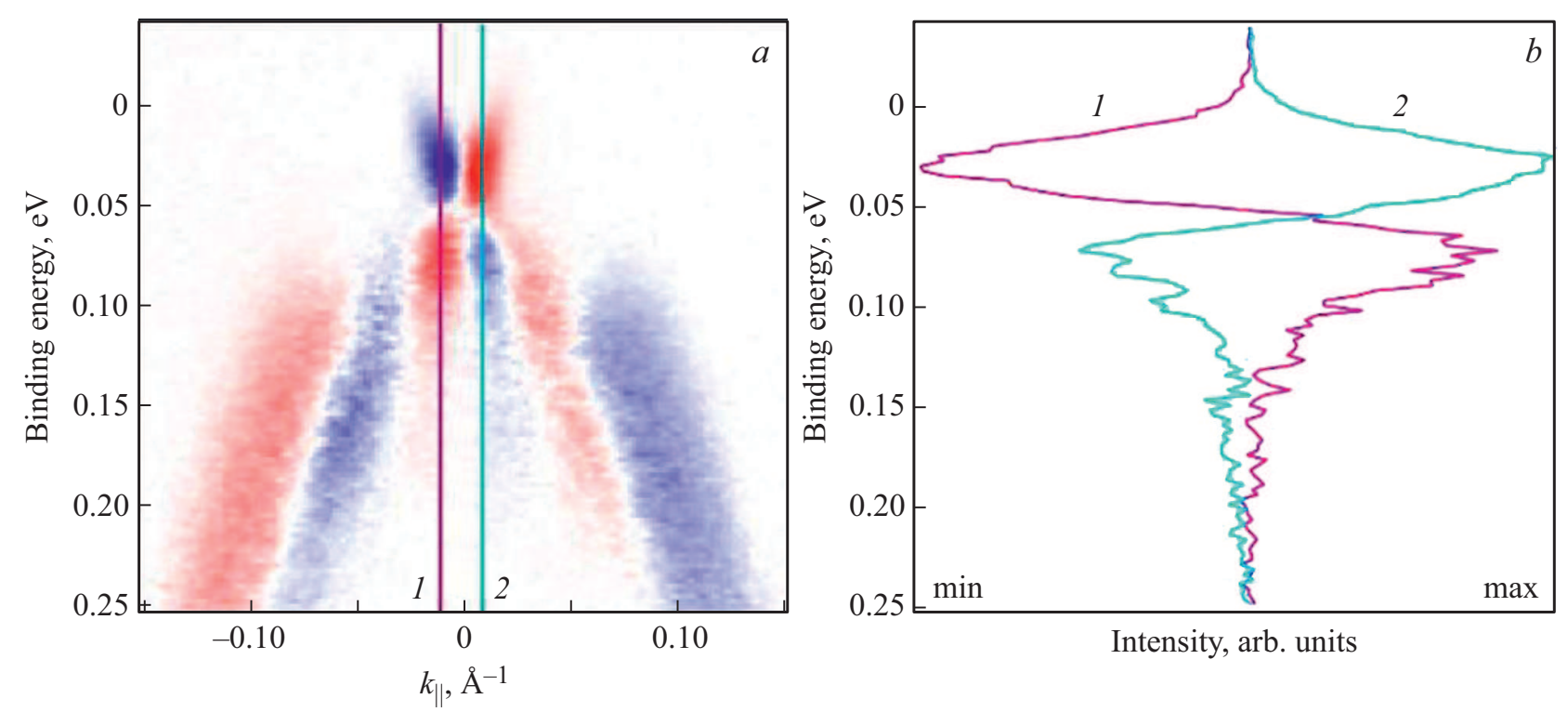

Рис. 4. $a-$ ФЭСУР-дисперсии дираковских состояний, полученных методом циркулярного дихроизма. Различной тональностью (слева и справа) показаны превалирующие вклады, соответствующие противоположным циркулярным поляризациям СИ. $b-$ Профили ФЭ-спектров, измеренные при $k_{\text {II }}$, отмеченных на $(a)$ вертикальными линиями 1 и 2.
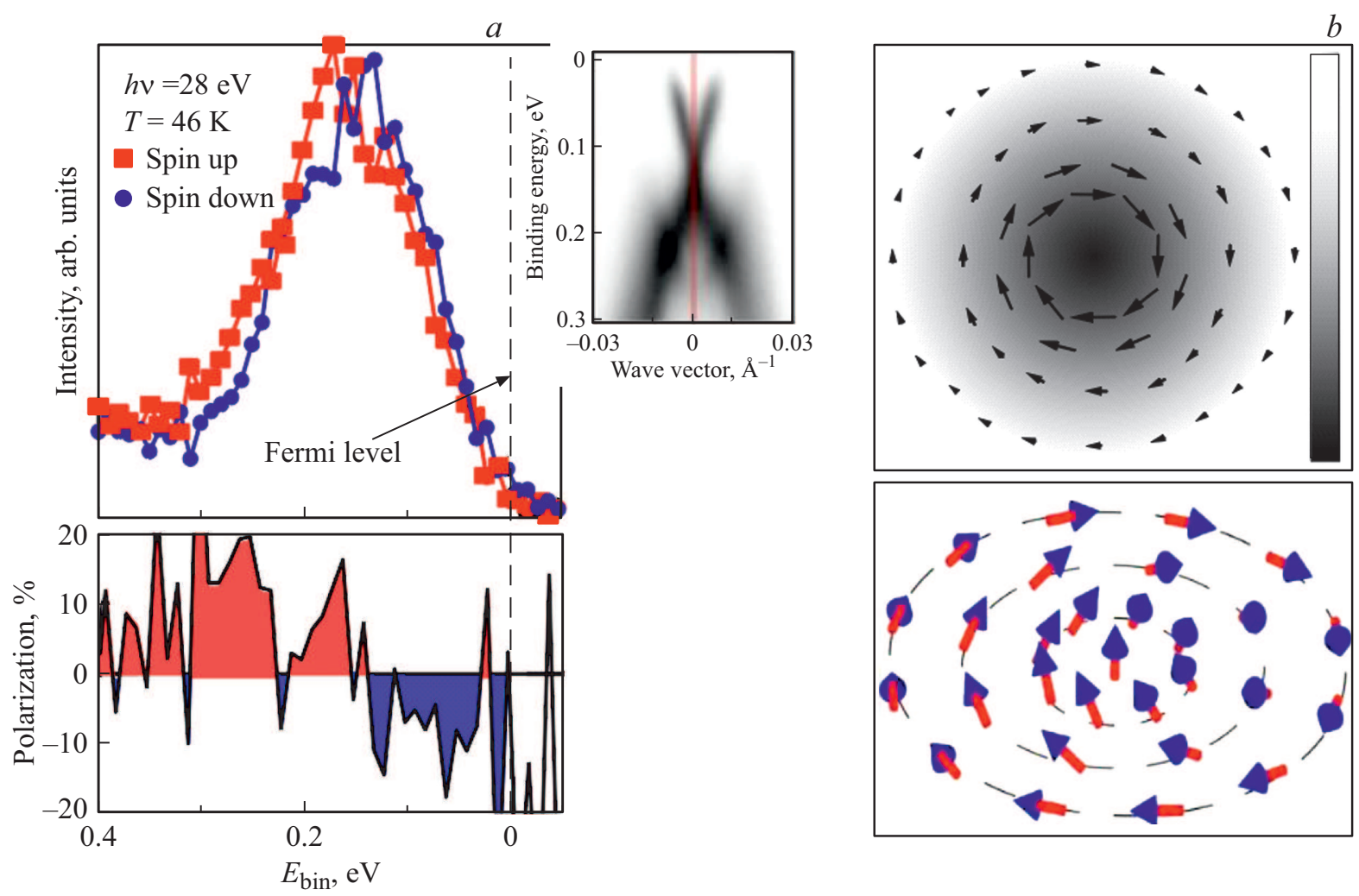

Рис. 5. $a$ - Фотоэлектронные спектры со спиновым разрешением для состояний дираковского конуса, измеренные в точке Дирака $\left(k_{\mathrm{II}}=0\right)$ для $\mathrm{Bi}_{1.09} \mathrm{Gd}_{0.06} \mathrm{Sb}_{0.85} \mathrm{Te}_{3}$ при энергии фотонов $28 \mathrm{eV}$ и температуре $46 \mathrm{~K}$ с использованием $p$-поляризованного синхротронного излучения. Спектры представлены в виде разложения на вклады, обусловленные электронными состояниями со спином вверх и спином вниз (квадратные и круглые символы). На нижней панели представлена вариация нормальной составляющей спиновой поляризации для различных энергий связи в области нижнего и верхнего дираковского конуса. В качестве вставки на панели (a) показана дисперсионная зависимость дираковских состояний, измеренная методом ФЭСУР, для сравнения. $b$ - Схематическое представление спиновой текстуры формируемой в области локального магнитного момента на магнитной атоме в матрице топологического изолятора с вариацией направления магнитного момента от центра у периферии (вид сверху и сбоку). 
излучения. На вкладыше показана соответствующая дисперсия дираковских состояний, измеренная методом ФЭСУР, для сравнения. На верхней панели представлены вклады, обусловленные электронными состояниями со спином вверх и спином вниз (квадратные и круглые символы соответственно). На нижней панели показана энергетическая зависимость результирующей спиновой поляризации по нормали к поверхности при вариации энергии связи, соответствующей переходу между состояниями верхнего и нижнего конуса дираковских состояний. Представленные спектры показывают явную инверсию спиновой поляризации между верхним и нижним конусом, характерную для магнитных ТИ, см., например [12-16]. Такая инверсная спиновая поляризации соответствует формированию „hedgehog-like“ спиновой текстуры с противоположной спиновой поляризацией состояний на противоположных краях щели, открываемой в точке Дирака, при $k_{\mathrm{II}}=0$, с постепенным поворотом спина параллельно поверхности при увеличении $k_{\mathrm{II}}[12]$. При этом, так как данная спиновая структура наблюдается при температуре значительно выше $T_{H}$, то это может являться дополнительным доказательством открытия щели в точке Дирака выше $T_{H}$. Несмотря на то, что данная температура выше температуры объемного дальнодействующего магнитного разупорядочения, наблюдаемая спиновая структура подобна той, которая обусловлена магнитным взаимодействием в ТИ.

Подводя общий основной итог всех представленных выше экспериментальных результатов можно сделать основной вывод о том, что в электронной и спиновой структуре Gd-допированного ТИ, измеряемой методом ФЭСУР, в области точки Дирака наблюдается открытие энергетической щели, причем, как выше, так и ниже температуры дальнодействующего магнитного упорядочения $\left(T_{H}\right)$. При этом величина открываемой щели в точке Дирака практически не меняется при переходе через $T_{H}$, но может модулироваться изменением поляризации СИ, используемого при ФЭСУР измерениях. Наблюдаемая при этом спиновая структура (измеренная методом ФЭСУР также выше $T_{H}$ ) подобна ,hedgehog-like“ спиновой текстуре, характерной для магнитного типа взаимодействий в ТИ. Эти экспериментальные наблюдения требуют анализа и соответствующего объяснения.

\section{3. Обсуждение результатов}

Мы предполагаем, что щель в точке Дирака, измеряемая методом ФЭСУР выше температуры нарушения дальнодействующего магнитного упорядочения $\left(T_{H}\right)$ появляется в спектрах в результате ФЭ-процесса при генерации дырки на $d, f$-валентных состояниях магнитного атома (в данном случае на атоме Gd) и формирования соответствующей спиновой текстуры вокруг генерируемой ФЭ-дырки, приводящей к взаимодействию (спариванию) дираковских фермионов в ТИ.
Стремясь экранировать генерируемую дырку окружающая среда (ТИ, характеризуемый высоким спин-орбитальным взаимодействием) индуцирует вокруг соответствующую спиновую текстуру с ротацией направления спинового момента, создаваемого дыркой на магнитном атоме (преимущественно направленным по нормали к поверхности, вследствие высокой анизотропии магнитного момента, характерного для магнитно-допированных топологических изоляторов [26]) к спиновой ориентации параллельно поверхности, характерной для поверхностных топологических состояний. Здесь мы рассматриваем только поверхностную область, где локализованы топологические поверхностные состояния, характеризующиеся дираковским конусом электронных состояний. Генерируемая спиновая текстура подобна текстуре магнитного вихревого скирмиона (vortex-skyrmion) [27,28] или мерона с вариацией спинового направления от перпендикулярного поверхности в центре к направлению параллельно поверхности на периферии [28]. При этом даже при температуре выше температуры дальнодействующего магнитного упорядочения спиновая ориентация параллельно поверхности на периферии генерируемого мерона жестко фиксируется соответствующим внутри-плоскостным направлением спинов топологических состояний. В результате этого имеет место также и фиксация спиновой ориентации в центре мерона перпендикулярно поверхности. Схематически подобная спиновая текстура с вариацией направления магнитного момента на величину $\pi / 2$ от центра к периферии показана на рис. $5, b$. В зависимости от магнитных свойств среды возможен дополнительно затухающий осциллирующий характер нормальной спиновой компоненты [29,30].

При этом следует отметить, что скирмионоподобные текстуры могут формироваться не только в магнитных металлах, но и в исходно немагнитных системах с усиленным спин-орбитальным взаимодействием вокруг атомов магнитных примесей на поверхности, как, например, для атомов $\mathrm{Fe}$ на поверхности $\mathrm{Au}(111)$ [29]. Исходя из чего, следует, что магнитно-допированные ТИ также могут являться благоприятной средой при определенных условиях для генерации скирмионоподобных спиновых текстур вокруг атомов магнитной примеси.

Мы предполагаем, что именно спиновая текстура скирмионоподобного (вернее мероноподобного) типа генерируется в процессе фотоэмиссии вокруг дырки на магнитном атоме в ТИ и взаимодействует с Дираковскими фермионами с противоположным импульсом и спиновой ориентацией, приводя к их частичной локализации в области генерируемой дырки. Данный процесс сопровождается „спариванием“ дираковских фермионов (частичной гибридизацией в области создаваемой дырки на магнитном атоме) непосредственно в течение ФЭ-процесса и открытием щели в точке Дирака в измеряемых ФЭСУР-дисперсиях. Данная спиновая текстура аналогична спиновой текстуре, создаваемой магнитной примесью в топологическом изоляторе, представленной в работе [30], и которая индуцируется с необходимостью 
для экранирования генерируемого фотоэмиссией локального магнитного момента на магнитном атоме внутри топологического изолятора. Только в рассматриваемом нами процессе подобная спиновая текстура формируется в результате генерации ФЭ-дырки на магнитном атоме выше температуры Нееля непосредственно в течение ФЭ-процесса, что и приводит к формированию щели в точке Дирака в $Ф Э$-спектрах выше температуры Нееля.

Представленный механизм отличается существенно от идеи появления немагнитной щели исключительно за счет эффектов „непересечения“ электронных состояний (avoided-crossing effect) при пересечении ветвей дираковского конуса с $d$-состояниями магнитного атома в области точки Дирака [17-19] независимо от температуры. В результате в точке Дирака должна формироваться гибридизационная щель, не имеющая магнитной природы. Однако, механизм, предлагаемый в данных работах, не может объяснить формирование щели в точке Дирака для $\mathrm{Bi}_{1.09} \mathrm{Gd}_{0.06} \mathrm{Sb}_{0.85} \mathrm{Te}_{3}$ по следующим причинам. С одной стороны для Gd-допированного топологического изолятора не формируется примесная $d$-зона, которая могла бы пересекать область дираковских состояний в точке Дирака и приводить к формированию соответствующей гибридизационной щели. Эксперименты по резонансной фотоэлектронной спектроскопии $[7,8]$ не показывают наличия каких-либо соответствующих $d$-зон в области точки Дирака. С другой стороны, как показали эксперименты по напылению калия, представленные на рис. 2, величина щели, измеряемая фотоэмиссией, не изменяется при вариации энергетического положения точки Дирака относительно уровня Ферми, что должно существенно влиять на условия и результат соответствующих эффектов „непересечения“ электронных состояний. В то же время, как показано на рис. 3, величина расщепления между дираковскими состояниями в точке Дирака изменяется при изменении поляризации синхротронного излучения, что свидетельствует о динамической природе измеряемой щели, обусловленной влиянием фотоэмиссионного процесса.

Как уже отмечалось, в рамках предлагаемого нами механизма открытие щели в точке Дирака выше $T_{H}$ происходит за счет „спаривания“ дираковских фермионов в области точки Дирака посредством их взаимодействия (смешивания) со спиновой текстурой, формируемой на дырке на магнитном атоме в процессе фотоэмиссии. Данный механизм в некоторой степени похож на механизм спаривания дираковских фермионов в эффектах индуцированной и топологической сверхпроводимости в системах на основе топологических изоляторов с формированием энергетической щели на уровне Ферми (от 1 и до $15 \mathrm{meV})$, наблюдаемой в ФЭСУР-дисперсиях [31-34], когда спаривание происходит посредством взаимодействия через фонон (или другие квазичастицы - поляроны, магноны и т.п.). Однако в рассматриваемом нами эффекте открытия щели в точке Дирака в магнитном топологическом изоляторе „спаривание“ дираковских фермионов должно происходить не через квазичастицы типа фонона, а посредством взаимодействия через квазичастицу магнитного характера типа магнитного вихря или скирмиона (мерона), т. е. щель должна формироваться не на уровне Ферми, а в точке Дирака, независимо от энергетического положения точки Дирака. При этом спиновая текстура формируемой магнитной квазичастицы должна содержать как компоненты со спиновой ориентаций перпендикулярно поверхности (out-of-plane spin orientation), которая обеспечивает возможность открытия щели в точке Дирака, так и компоненты со спиновой ориентацией вдоль поверхности (in-plane spin orientation) с ориентацией спина перпендикулярно импульсу электрона, характерной для топологических поверхностных состояний, которые обеспечивают возможность взаимодействия с дираковскими фермионами, т.е. как раз подобно спиновой текстуре вихревого скирмиона или мерона.

В работах $[27,28]$ анализировались эффекты индуцированного взаимодействия (proximity effect) дираковских поверхностных состояний ТИ с магнитными скирмионами, формируемыми в тонких металлических и диэлектических слоях на поверхности топологического изолятора. Было показано, что данное взаимодействие приводит к локализации дираковских состояний (electron confinement) и формированию связанных состояний на поверхности топологического изолятора, а также к появлению в гамильтониане для дираковских состояний „массового“ или магнитного члена, открывающего щель в точке Г. При этом вид данного „массового“ члена и его влияние определяются магнитной текстурой скирмиона и эффективной спиновой поляризацией перпендикулярно поверхности, генерируемой скирмионом.

Предлагаемый механизм открытия щели (или „приобретения массы“) подобен в некоторой степени механизму Хиггса обретения массы у элементарных частиц. В соответствии с этим механизмом появление массы у элементарных частиц обусловлено взаимодействием с бозонным полем Хиггса. В твердом теле проводниками такого взаимодействия могут быть фононы, поляроны и др. квазичастицы, взаимодействие с которыми обуславливает, например, спаривание дираковских фермионов в явлениях топологической сверхпроводимости с формированием соответствующей запрещенной зоны на уровне Ферми [34,35]. В работе [36] отмечалась возможность использования механизма, подобного механизму Хиггса для объяснения появления щели в точке Дирака в ТИ $\mathrm{TlBi}\left(\mathrm{S}_{1-x} \mathrm{Se}_{x}\right)_{2}$. В данной работе появление запрещенной зоны носило спонтанный характер при параметре , $x^{\text {“ }}=0.5$. Таким образом, возможно существование некого аналога механизма Хиггса в твердом теле в котором частицы обретают массу ввиду спонтанного локального нарушения симметрии. В нашей работе мы предполагаем, что „спаривание“ электронных состояний с противоположных ветвей дираковского конуса обусловлено их взаимодействием через магнитную квазичастицу с мероноподобной спиновой текстурой, 
локализуемую в области магнитного атома. Данная генерируемая фотоэмиссией динамическая магнитная квазичастица связывает дираковские фермионы с противоположным импульсом и спиновой ориентацией, приводя к обретению массы и формированию уже „массивных“ фермионов вследствие данной их частичной локализации. Именно эта частичная локализация взаимодействия между дираковскими фермионами в области формируемой (короткоживущей) ФЭ-дырки с соответствующим нарушением симметрии обращения времени и обеспечивает формирование щели в точке Дирака в течение ФЭ-процесса (т.е. обретение массы для безмассовых дираковских фермионов).

Что касается величины щели, открываемой в точке Дирака, то в соответствии с анализом, проведенным в работе [30] одиночная магнитная примесь или разупорядоченные магнитные примеси также могут модифицировать электронную структуру ТИ и открывать локальную щель в точке Дирака, непосредственно в области локализации магнитных примесных атомов. В работе [37] данное предположение было экспериментально подтверждено методом сканирующей туннельной микроскопии и спектроскопии. Было показано, что для магнитно-допированного топологического изолятора $\mathrm{Cr}_{0.08}\left(\mathrm{Bi}_{0.1} \mathrm{Sb}_{0.9}\right)_{1.92} \mathrm{Te}_{3}$ энергетическая щель в точке Дирака действительно открывается в области локализации магнитного атома. При этом для данной концентрации магнитного металла среднее значение открываемой щели было определено на уровне $25-30 \mathrm{meV}$. Что в некоторой степени коррелирует с величиной щели, определенной для исследуемой нами системы ниже $T_{H}$, исходя из рис. 2, принимая во внимание приблизительно эквивалентный уровень магнитного допирования. Интегральная по поверхности щель в точке Дирака в общей электронной структуре магнитного ТИ формируется, когда вступают в силу эффекты дальнодействующего магнитного взаимодействия между магнитными моментами ближайших магнитных атомов при их ориентации по нормали к поверхности (ниже $T_{H}$ ). При этом формируемое усредненное магнитное поле и величина эффективного магнитного момента, открывающего интегральную щель в точке Дирака, определяются в основном величиной атомного магнитного момента (для Gd-допированного ТИ (см. работы [5-8])).

Это позволяет предположить, что эффективный магнитный момент спиновой текстуры, индуцируемой на отдельном магнитном (Gd) атоме в процессе фотоэмиссии, не сильно отличается от усредненного магнитного момента в пересчете на один атом для объемного магнитно-допированного ТИ. Поэтому величина щели в точке Дирака при измерениях методом ФЭСУР выше $T_{H}$ не должна значительно измениться по сравнению с измерениями ниже $T_{H}$, что и наблюдается в эксперименте. Меняется только интенсивность дираковских состояний в ФЭ-спектрах. При этом индуцированная мероноподобная спиновая текстура в ТИ с периферийной спиновой ориентаций вдоль поверхности ориентирует спиновый момент в центре текстуры перпендикулярно поверхности в том числе и выше температуры Нееля. Это и обуславливает наблюдение щели в точке Дирака в ФЭ-эксперименте выше $T_{H}$.

При этом, генерируемая фотоэмиссией спиновая текстура уже не определяется эффектами дальнодействующего магнитного упорядочения. До тех пор пока температурное уширение остается меньше величины щели в точке Дирака, генерируемой фотоэмиссией, условия для „спаривания“ дираковских фермионов посредством генерируемой спиновой текстуры, сохраняются. Поэтому щель в точке Дирака, обусловленная данным процессом, видна выше $T_{H}$, несмотря на отсутствие дальнодействующего магнитного порядка. При этом природа щели в точке Дирака, генерируемой фотоэмиссией, остается магнитной, так как в формируемом взаимодействии важную роль играет мероноподобная спиновая текстура, генерируемая в области магнитного атома при фотоэмиссии, которая определяется магнитными свойствами исследуемой системы.

Необходимость наличия эффективного магнитного момента, создаваемого генерируемой спиновой текстурой в области ФЭ-дырки, подтверждается отсутствием видимой щели в экспериментальных ФЭСУР-дисперсиях в точке Дирака для чистых (немагнитных) ТИ по сравнению с магнитно-допированными.

При этом динамическая щель, открываемая в точке Дирака при фотовозбуждении, может также определять и соответствующие динамические магнитные и магнитно-транспортные характеристики. В частности, формирование магнитного момента, наблюдаемого в XMCD измерениях при фотовозбуждении остовных уровней магнитных атомов для РЗМ-допированных топологических изоляторов при температурах значительно выше температуры магнитного упорядочения [5-8] также могут быть связанными с описанными выше эффектами. Можно также предположить возможность реализации ФЭ-индуцированного QАНЕ при повышенных температурах с возможностью модулирования эффекта поляризацией и энергией возбуждающего излучения.

Аналогичные эффекты формирования щели в точке Дирака в измеряемых ФЭСУР дисперсионных зависимостях выше температуры Нееля наблюдаются и для других АФМ ТИ. В частности, для магнитно-упорядоченного АФМ ТИ со стехиометрией $\mathrm{MnBi}_{2} \mathrm{Te}_{4}$ щель в точке Дирака в измеряемых ФЭ-спектрах сохраняется выше $T_{H}[9,11]$, что также может быть связано с эффектами ФЭ-индуцированного „спаривания“ дираковских фермионов и формированием динамической щели в точке Дирака.

Для более детального анализа зависимости величины щели в точке Дирака от температуры, механизма ее формирования и взаимосвязи со спиновой текстурой, генерируемой в процессе фотоэмиссии, требуются дальнейшие более детальные исследования спиновой структуры магнитно-допированных топологических изоляторов в 
зависимости от температуры и энергии фотонов, в том числе с использованием других методов исследования.

\section{4. Заключение}

Методами фотоэлектронной спектроскопии с угловым разрешением проведены детальные исследования электронной структуры в области точки Дирака для магнитно-допированного ТИ со стехиометрией $\mathrm{Bi}_{1.09} \mathrm{Gd}_{0.06} \mathrm{Sb}_{0.85} \mathrm{Te}_{3}$ при различных температурах (выше и ниже температуры Нееля) и различных поляризациях СИ. Показано, что точка Дирака для данного материала располагается вблизи уровня Ферми. Путем анализа дисперсий поверхностных топологических состояний методом ФЭСУР выявлена энергетическая щель 25-35 meV (в зависимости от условий измерений), открываемая в точке Дирака, которая остается открытой выше температуры Нееля, когда дальнодействующий магнитный порядок в системе уже нарушается. Измерения магнитных свойств методом сверхпроводящей магнитометрии (СКВИД) показали АФМ-порядок магнитного упорядочения по нормали к поверхности с температурой Нееля для перехода между АФМ- и ПМ-фазой в объеме, равной $8.3 \mathrm{~K}$. Исследования температурной зависимости интенсивности пика состояний в области точки Дирака в ФЭСУР-спектрах подтвердили наличие поверхностного АФМ/ПМ-перехода при температурах $10-15 \mathrm{~K}$ и показали возможность индикации магнитного перехода методами ФЭСУР непосредственно в процессе ФЭ-эксперимента. Более детальный анализ величины расщепления между состояниями верхнего и нижнего дираковского конуса (т.е. величины энергетической щели) в точке Дирака в измеряемых ФЭСУР дисперсиях показал зависимость щели в точке Дирака от типа поляризации СИ $(28-30 \mathrm{meV}$ для р-поляризации и $22-25 \mathrm{meV}$ для циркулярно-поляризованного излучения противоположной хиральности). В работе предложен механизм открытия щели в точке Дирака выше температуры Нееля вследствие „спаривания“ дираковских фермионов с противоположным импульсом и спиновой ориентацией в результате из взаимодействия со спиновой текстурой, формируемой в ТИ непосредственно в процессе фотоэмисии в области фотоэмиссионной дырки на атоме магнитной примеси $(\mathrm{Gd})$. Т. е щель в точке Дирака, измеряемая методом ФЭСУР выше температуры Нееля, является динамической и формируется непосредственно в процессе фотоэмиссии. При этом природа щели остается магнитной (хотя и не определяется наличием дальнодействующего магнитного порядка) и обусловлена магнитными свойствами магнитного топологического изолятора, что и определяет практически неизменность величины щели при переходе через температуру Нееля. Подтверждением динамического характера генерируемой щели является зависимость ее величины от поляризации синхротронного излучения.

\section{Финансирование работы}

Работа выполнена в рамках финансовой поддержки по гранту на НИР Санкт-Петербургского государственного университета (Pure 1D 40990069) и гранта РНФ № 18-12-00062.

\section{Конфликт интересов}

У авторов нет конфликта интересов.

\section{Список литературы}

[1] J. Wang, B.Lian, X.-L. Qi, S.-C. Zhang, Phys. Rev. B 92, 081107(R) (2015).

[2] Y.L. Chen, J.-H. Chu, J.G. Analytis, Z.K. Liu, K. Igarashi, H.-H. Kuo, X.L. Qi, S.K. Mo, R.G. Moore, D.H. Lu, M. Hashimoto, T. Sasagawa, S.C. Zhang, I.R. Fisher, Z. Hussain, Z.X. Shen. Science 329, 659 (2010).

[3] C.-Z. Chang, J. Zhang, X. Feng, J. Shen, Z. Zhang, M. Guo, K. Li, Y. Ou, P. Wei, L.-L. Wang, Z.-Q. Ji, Y. Feng, S. Ji, X. Chen, J. Jia, X. Dai, Z. Fang, S.-C. Zhang, K. He, Y. Wang, L. Lu, X.-C. Ma, Q.-K. Xue. Science 340, 167 (2013).

[4] C.-Z. Chang, W. Zhao, D.Y. Kim, H. Zhang, B.A. Assaf, D. Heiman, S.-C. Zhang, C. Liu, M.H.W. Chan, J.S. Moodera. Nature Mater. 14, 473 (2015).

[5] J. Kim, E.-H. Shin, M.K. Sharma, K. Ihm, O. Dugerjav, C. Hwang, H. Lee, K.-T. Ko, J.-H. Park, M. Kim, H. Kim, M.-H. Jung. Sci. Rep. 9, 1331 (2019).

[6] S.-R. Jian, P.H. Le, C.-W. Luo, J.-Y. Juang. J. Appl. Phys. 121, 175302 (2017).

[7] A.M. Shikin, D.A. Estyunin, Yu.I. Surnin, A.V. Koroleva, E.V. Shevchenko, K.A. Kokh, O.E. Tereshchenko, S. Kumar, E.F. Schwier, K. Shimada, T. Yoshikawa, Y. Saitoh, Y. Takeda, A. Kimura. Sci. Rep. 9, 4813 (2019).

[8] С.О. Фильнов, Ю.А. Сурнин, А.В. Королева, И.И. Климовских, Д.А. Естюнин, А.Ю. Варыхалов, К.А. Бокай, К.А. Кох, О.Е. Терещенко, В.А. Голяшов, Е.В. Шевченко, А.М. Шикин. ЖЭТФ 3, 483, (2019).

[9] M.M. Otrokov, I.I. Klimovskikh, H. Bentmann, A. Zeugner, Z.S. Aliev, S. Gass, A.U.B. Wolter, A.V. Koroleva, D.A. Estyunin, A.M. Shikin, M. Blanco-Rey, M. Hoffmann, A.Yu. Vyazovskaya, S.V. Eremeev, Y.M. Koroteev, I.R. Amiraslanov, M.B. Babanly, N.T. Mamedov, N.A. Abdullayev, V.N. Zverev, B. Büchner, E.F. Schwier, S. Kumar, A. Kimura, L. Petaccia, G. Di Santo, R.C. Vidal, S. Schatz, K.Kißner, C.-H. Min, S.K. Moser, T.R.F. Peixoto, F. Reinert, A. Ernst, P.M. Echenique, A. Isaeva, E.V. Chulkov. ArXiv: 1809, 07389 (2018).

[10] D. Zhang, M. Shi, T. Zhu, D. Xing, H. Zhang, J. Wang. Phys. Rev. Lett. 122, 206401 (2019).

[11] S.H. Lee, Y. Zhu, Y. Wang, L. Miao, T. Pillsbury, S. Kempinger, D. Graf, N. Alem, C.-Z. Chang, N. Samarth, Z. Mao. ArXiv:1812.00339 (2019).

[12] S.-Y. Xu, M. Neupane, C. Liu, D. Zhang, A. Richardella, L.A. Wray, N. Alidoust, M. Leandersson, T. Balasubramanian, J. Sánchez-Barriga, O. Rader, G. Landolt, B. Slomski, J.H. Dil, J. Osterwalder, T-R. Chang, H-T. Jeng, H. Lin, A. Bansil, N. Samarth, M.Z. Hasan. Nature Phys. 8, 616 (2012). 
[13] Y.L. Chen, J.-H. Chu, J.G. Analytis, Z.K. Liu, K. Igarashi, H.-H. Kuo, X.L. Qi, S.K. Mo, R.G. Moore, D.H. Lu, M. Hashimoto, T. Sasagawa, S.C. Zhang, I.R. Fisher, Z. Hussain, Z.X. Shen. Science 329, 659 (2010).

[14] A.M. Shikin, A.A. Rybkina, D.A. Estyunin, D.M. Sostina, I.I. Klimovskikh, V.Yu. Voroshnin, A.G. Rybkin, K.A. Kokh, O.E. Tereshchenko, L. Petaccia, G. Di Santo, A. Kimura, P.N. Skirdkov, K.A. Zvezdin, A.K. Zvezdin. Sci. Rep. 8, 6544 (2018).

[15] A.M. Shikin, A.A. Rybkina, D.A. Estyunin, D.M. Sostina, V.Yu. Voroshnin, I.I. Klimovskikh, A.G. Rybkin, Yu.A. Surnin, K.A. Kokh, O.E. Tereshchenko, L. Petaccia, G. Di Santo, P.N. Skirdkov, K.A. Zvezdin, A.K. Zvezdin, A. Kimura, E.V. Chulkov, E.E. Krasovskii. Phys. Rev. B 97, 245407 (2018).

[16] A.M. Shikin, A.A. Rybkina, I.I. Klimovskikh, M.V. Filianina, K.A. Kokh, O.E. Tereshchenko, P.N. Skirdkov, K.A. Zvezdin, A.K. Zvezdin, Appl. Phys. Lett. 109, 222404 (2016).

[17] J. Sánchez-Barriga, A. Varykhalov, G. Springholz, H. Steiner, R. Kirchschlager, G. Bauer, O. Caha, E. Schierle, E. Weschke, A.A. Ünal, S. Valencia, M. Dunst, J. Braun, H. Ebert, J. Minár, E. Golias, L.V. Yashina, A. Ney, V. Holý, O. Rader. Nature Commun. 7, 10559 (2015).

[18] A.M. Black-Schaffer, A.V. Balatsky, J. Fransson. Phys. Rev. B 91, 201411(R) (2015).

[19] Y. Xu, J. Chiu, L. Miao, H. He, Z. Alpichshev, A. Kapitulnik, R.R. Biswas, L. Andrew Wray. Nature Commun. 8, 14081 (2016).

[20] G. Rosenberg, M. Franz. Phys. Rev. B 85, 195119 (2012).

[21] C.-Z. Chang, M. Li. J. Phys.: Condens. Matter 28, 123002 (2016).

[22] M.G. Vergniory, M.M. Otrokov, D. Thonig, M. Hoffmann, I.V. Maznichenko, M. Geilhufe, X. Zubizarreta, S. Ostanin, A. Marmodoro, J. Henk, W. Hergert, I. Mertig, E.V. Chulkov, A. Ernst. Phys. Rev. B 89, 165202 (2014).

[23] M. Bode, M. Getzlaff, A. Kubetzka, R. Pascal, O. Pietzsch, R. Wiesendanger. Phys. Rev. Lett. 83, 3017 (1999).

[24] A.V. Fedorov, K. Starke, G. Kaindl, Phys. Rev. B 50, 2739 (1994).

[25] A. Chikina, M. Hoeppner, S. Seiro, K. Kummer, S. Danzenbaecher, S. Patil, A. Generalov, M. Guettler, Yu. Kucherenko, E.V. Chulkov, Yu.M. Koroteev, K. Koepernik, C. Geibel, M. Shi, M. Radovic, C. Laubschat, D.V. Vyalikh. Nature Commun. 5, 3171 (2014).

[26] A.S. Nunez, J. Fernandez-Rossier. Solid State Commun., 152, 403 (2012).

[27] D. Andrikopoulos, B. Sorée, J.D. Boeck. J. Appl. Phys. 119, 193903 (2016).

[28] F. Loder, A.P. Kampf, T. Kopp, D. Braak. Phys. Rev. B 96, 024508 (2017).

[29] S. Lounis, A. Bringer, S. Bluegel, Phys. Rev. Lett. 108, 207202 (2012).

[30] Q. Liu, C.-X. Liu, C. Xu, X.-L. Qi, S.-C. Zhang. Phys. Rev. Lett. 102, 156603 (2009).

[31] L. Andrew Wray, Su-Yang Xu, Yuqi Xia, Yew San Hor, Dong Qian, Alexei V. Fedorov, Hsin Lin, Arun Bansil, Robert J. Cava\&ं M. Zahid Hasan, Nature Phys. 6, 855 (2010).

[32] G. Du, J. Shao, X. Yang, Z. Du, D. Fang, J. Wang, K. Ran, J. Wen, C. Zhang, H. Yang, Y. Zhang, H-H. Wen. Nature Commun. 8, 14466 (2016).
[33] E. Wang, H. Ding, A.V. Fedorov, W. Yao, Z. Li, Y.-F. Lv, K. Zhao, L.-G. Zhang, Z. Xu, J. Schneeloch, R. Zhong, S.H. Ji, L. Wang, K. He, X. Ma, G. Gu, H. Yao, Q.-K. Xue, X. Chen, S. Zhou. Nature Phys. 9, 621 (2013).

[34] S.-Y. Xu, N. Alidoust, I. Belopolski, A. Richardella, C. Liu, M. Neupane, G. Bian, S-H. Huang, R. Sankar, C. Fang, B. Dellabetta, W. Dai, Q. Li, M.J. Gilbert, F. Chou, N. Samarth, M.Z. Hasan. Nature Phys. 10, 943 (2014).

[35] P. Zhang, K. Yaji, T. Hashimoto, Y. Ota, T. Kondo, K. Okazaki, Z. Wang, J. Wen, G.D. Gu, H. Ding, S. Shin. Sci. 360, 182 (2018).

[36] T. Sato, Kouji Segawa, K. Kosaka, S. Souma, K. Nakayama, K. Eto, T. Minami, Yoichi Ando \& T. Takahashi. Nature Phys. 7, 840 (2011).

[37] I. Lee, C.K. Kim, J. Lee, S.J.L. Billinge, R. Zhong, J.A. Schneeloch, T. Liu, T. Valla, J.M. Tranquada, G. Gu, J.C. Séamus Davis. PNAS 112, 1316 (2015).

Редактор К.В. Емцев 